\title{
SEISMIC DETECTION OF BOUNDARIES OF STELLAR CONVECTIVE REGIONS
}

\author{
MÁRIO J.P.F.G. MONTEIRO \\ DMA-FCUP and CAUP, Universidade do Porto, Portugal \\ JØRGEN CHRISTENSEN-DALSGAARD \\ TAC and Inst. Fysik og Astronomi, Aarhus Universitet, Denmark \\ AND \\ MICHAEL J. THOMPSON \\ Astronomy Unit, QMW, University of London, England
}

The edge of a convective region inside a star gives rise to a characteristic periodic signal in the frequencies of its global p-modes (e.g. [1], [4]), such that the frequencies $\omega$ are then essentially a smooth function of the mode order $n$ plus a periodic component $\delta \omega_{p}=A(\omega) \cos \left[2\left(\omega \bar{\tau}_{\mathrm{d}}+\phi_{0}\right)\right]$. Here the amplitude is $A(\omega)=\sqrt{A_{1}^{2} / \omega^{4}+A_{2}^{2} / \omega^{2}}$, with $A_{1}$ and $A_{2}$ being values that depend weakly on frequency $\omega: A_{1}$ is always present in general, but $A_{2}$ will be non-zero only if there is overshoot; $\bar{\tau}_{d}$ is essentially the acoustical depth $\tau$ (i.e. the sound travel time) of the edge of the convection zone measured from the surface of the star; and $\phi_{0}$ is a constant related to the phase of the eigenfunctions. To facilitate the comparison between different stars, we consider the amplitude evaluated at a fiducial frequency by defining $A_{\mathrm{d}} \equiv A(\tilde{\omega})$. For the Sun, we chose as the reference frequency $\tilde{\omega}=2500 \mu \mathrm{Hz}$. If we take this value and scale it for other stars (using just a standard homology scaling for frequencies), we find $\tilde{\omega} / 2 \pi=2500 \mu \mathrm{Hz} \times\left(M / M_{\odot}\right)^{1 / 2}\left(R / R_{\odot}\right)^{-3 / 2}$.

Concentrating on the case without convective overshoot, we have

$$
A_{\mathrm{d}}=\frac{A_{1}}{\tilde{\omega}^{2}} \propto \frac{g}{\tilde{\omega}^{2} \tau_{\mathrm{t}}} \frac{\mathrm{d} \nabla_{\mathrm{r}}}{\mathrm{d} \tau}
$$

where $\tau_{\mathrm{t}}$ is the total acoustical radius of the star, and gravitational acceleration $g$ and radiative temperature gradient $\nabla_{\mathbf{r}}$ are evaluated at the convective boundary. This gives the dependence of the amplitude $A_{\mathrm{d}}$ on the position of the boundary. To study the variation of $A_{\mathrm{d}}$ with stellar mass we need to know how the different functions of the stellar structure in equation (1) depend on the mass. Assuming that opacity $\kappa \propto \rho^{\alpha} T^{-\beta}$, an homology scaling gives $\mathrm{d} \nabla_{\mathrm{r}} / \mathrm{d} \tau \propto L R^{-3 \alpha+\beta-3 / 2} M^{-5 / 2-\beta+\alpha}$. Adopting $\alpha=1$ and $\beta=7 / 2$, we find that

$$
A_{\mathrm{d}}=A_{\mathrm{d}, \odot}\left(L / L_{\odot}\right)\left(R / R_{\odot}\right)^{-1}\left(M / M_{\odot}\right)^{-5},
$$

where we have used as reference the value $A_{\mathrm{d}, \odot}$ found for the Sun. 
The feasibility of detecting the signal is measured by the dimensionless ratio $A_{\mathrm{d}} / \tilde{\omega}=3.5 \times 10^{-5}\left(L / L_{\odot}\right)\left(R / R_{\odot}\right)^{5 / 2}\left(M_{\odot} / M\right)^{-11 / 2}$. Thus the signal we seek in the frequencies is typically a few parts in $10^{5}$.

For stars of mass similar to the Sun $\left(0.8 \lesssim M / M_{\odot} \lesssim 1.2\right)$ we have roughly that $R \propto M^{1.5}$ and $L \propto M^{5}$ (Figs 22.2, 22.3 of [2]); thus the amplitude of the signal and its ratio to the reference frequency scale as

$$
A_{\mathrm{d}}=A_{\mathrm{d}, \odot}\left(M / M_{\odot}\right)^{-1.5} \quad \text { and } \quad A_{\mathrm{d}} / \tilde{\omega}=3.5 \times 10^{-5}\left(M / M_{\odot}\right)^{-5 / 4}
$$

Fig. 1: Expected dependence on stellar mass of the amplitude of the periodic signal in the frequencies arising from the edge of a convective envelope (open circles) or from the edge of a convective core (triangles), as predicted by equation (1). Also shown are values from expressions (2) (dashed line) and (3) (dotted line) for the expected amplitude. The measured values for the signal in the frequencies (with degree $\leq 4)$ are represented by the filled circles.

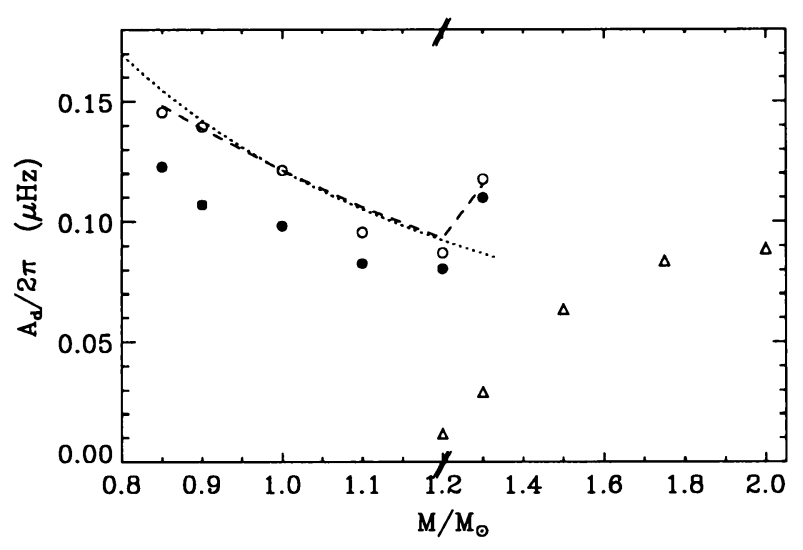

From the p-mode frequencies of ZAMS models for different stars we have determined the amplitude of the signal arising from edges of convective regions using the least-squares method described by [4]. The modes used have degrees $l$ between 0 and 4. These modes might in principle be observed for distant stars. The numerical results of the fits are shown in Figure 1. It is clear that the signal that one expects to detect from the edge of convective envelopes in stars of mass up to $1.3 M_{\odot}$ can indeed be measured in frequency data that we can hope to obtain for such stars. We have also calculated the amplitude of the signal that would be produced by the convective core of a star with mass $\gtrsim 1.5 M_{\odot}$; as shown by the figure this should also be large enough that it ought to be detectable.

Finally, we make two remarks. First we note that the predicted amplitudes in velocity $\left(v_{\text {osc }}\right)$ for solar-like oscillations in other stars (e.g. [3]), for this mass range, are expected to increase with mass according to $v_{\mathrm{osc}} \propto L / M \propto M^{4}$. Such a behaviour would compensate the decrease with mass found here for the detectability of the signal. Secondly, the composition inhomogeneity arising from evolution implies a strong increase in the detectability of the core for older higher-mass stars. This is expected to be important for studying high-mass stars with a large convective core and a negligible envelope; in particular, overshoot from convective cores may modify the signal in measurable ways.

[1] Christensen-Dalsgaard J., Monteiro M.J.P.F.G., Thompson M.J. (1995), MNRAS 276, 283

[2] Kippenhahn R., Weigert A. (1990), Stellar Structure and Evolution, Springer-Verlag

[3] Kjeldsen H., Bedding T.R. (1995), A\&A 293, 87

[4] Monteiro M.J.P.F.G., Christensen-Dalsgaard J., Thompson M.J. (1994), A\&A 283, 247 